Paidéia, 2001, 11(20), 39-47

\title{
MATURIDADE EMOCIONAL, LOCUS DE CONTROLE E ANSIEDADE EM PRÉ- ADOLESCENTES OBESOS ${ }^{1}$
}

Ana Maria Pimenta Carvalho ${ }^{2}$ EERP - Universidade de São Paulo

\begin{abstract}
RESUMO: Investigou-se aspectos do funcionamento psicológico como a maturidade emocional, locus de controle e ansiedade em pré-adolescentes obesos, através dos instrumentos: Teste do Desenho da Figura Humana (DFH), Escala de Locus de Controle para crianças e Escala de Ansiedade "RCMS". Os resultados não apontaram uma tendência específica quanto ao locus de controle ser mais interno ou externo. Em relação à ansiedade, enquanto traço de personalidade, a maioria dos sujeitos situou-se dentro do esperado para seu grupo etário. No DFH os índices relativos à Escala Evolutiva e à Escala Emocional encontraram-se também dentro do esperado, em sua maior parte. Tais resultados sugerem que não existe diferença entre pré-adolescentes obesos e pré-adolescentes em gera', quanto às variáveis estudadas. Entretanto, ser obeso pode estar associado a dificuldades de elaboração de aspectos evolutivos e emocionais para alguns indivíduos.
\end{abstract}

Palavras chave: obesidade; avaliação psicológica; pré-adolescentes

\section{EMOTIONAL MATURITY, LOCUS OF CONTROL AND ANXIETY IN OBESE PREADOLESCENTS}

\begin{abstract}
Aspects of psychological functioning such as emotional maturity, locus of control and anxiety had been evaluated in obese preadolescents. The following instruments were used: Draw a Person Test - DAT; Locus of Control Scale for Children and the Revised Children Manifest Anxiety Scale (Portuguese versions). Results show no tendency for the locus of control to be more external or internal, when compared to normative data. The scores of anxiety, as personality trait, are compatible to the normative data. The DAT analysis showed that most of the results related to Developmental and Emotional Scales are within the norm values. These results suggest no differences between obese preadolescents and preadolescents in general, related to the aspects approached in this study. They also suggest that, for some individuals, being obese may be associated to some emotional suffering and difficulties in dealing with developmental aspects.
\end{abstract}

Key words: obesity; psychological evaluation, preadolescents

Tem havido, nos últimos anos, um avanço acelerado da obesidade em toda a população. Entre crianças e adolescentes a proporção deste aumento é ainda maior. Segundo dados do Instituto Brasileiro de Geografia e Estatística (IBGE) e da Sociedade Brasileira de Pediatria, no ano de $1975,8 \%$ das mulheres eram obesas, $3 \%$ dos homens e $3 \%$ das crianças. Já no ano de 1997 estes números aumentaram para $13 \%$ entre as mulheres, $7 \%$ entre os homens $\mathrm{e}$ $15 \%$ entre as crianças, havendo portanto, um cresci-

\footnotetext{
${ }^{1}$ Artigo recebido para publicação em 06/2001; aceito em 10/2001 2 Endereço para correspondência: Ana Maria Pimenta Carvalho, Departamento de Enfermagem Psiquiátrica e Ciências Humanas, Escola de Enfermagem de Ribeirão Preto-USP, Av. Bandeirantes, 3900, Monte Alegre, Ribeirão Preto, SP, CEP 14040-90 I, E-mail anacar@eerp.usp.br
}

mento de $500 \%$ entre estas últimas. Entre os adolescentes o índice de obesidade é $25 \%$ (Folha de São Paulo, 1999).

A obesidade é uma situação de difícil controle, apresentando um alto percentual de insucessos terapêuticos e de sérias repercussões orgânicas e psicossociais ao longo do tempo. Assim, sua prevenção e controle devem ter início na infância (Escrivão \& Lopes, 1995).

Fisberg (1995) considera que, entre as alterações do nosso corpo, a obesidade é a mais complexa e de difícil entendimento, havendo a necessidade de uma abordagem multidisciplinar do problema. Neste sentido, Kahtalian (1992) argumenta que é preci- 
so abordar a fome em seu aspecto psicossocial, já que, juntamente com a sede, estas são as mais importantes forças motivadoras conhecidas. Assim, os aspectos psicológicos também devem ser levados em conta, visto que os casos de obesidade causados por patologias endócrinas ou genéticas bem definidas, constituem um percentual muito pequeno (Escrivão \& Lopes, 1995).

Andrade (1995) em um estudo no ambulatório de Obesidade Infantil da Universidade Federal de São Paulo, em 134 casos atendidos, verificou que $76,8 \%$ destas crianças apresentavam razões emocionais importantes, associadas ao surgimento e à evolução da obesidade. Assim, estudos que busquem entender as características psicológicas ligadas à obesidade são muito importantes.

Campos (1993) identificou as seguintes características psicológicas em adultos obesos por hiperfagia: passividade e submissão, preocupação excessiva com comida, ingestão compulsiva de alimentos e drogas, dependência e infantilização, primitivismo, não aceitação do esquema corporal, temor de não ser aceito ou amado, indicadores de dificuldades de adaptação social, bloqueio da agressividade, dificuldade para absorver frustração, desamparo, insegurança, intolerância e culpa. No que diz respeito às crianças obesas, esta autora afirma ainda que elas são mais regredidas e infantilizadas; tendo dificuldades de lidar com suas experiências de forma simbólica, de adiar satisfações e obter prazer nas relações sociais, de lidar com a sexualidade, além de uma baixa auto-estima e dependência materna.

Kahtalian (1992) considera que o ato de comer, para os obesos, é tido como tranqüilizador, como uma forma de localizar a ansiedade e a angústia no corpo, sendo apresentadas também dificuldades de lidar com a frustração e com os limites.

Portanto, segundo a literatura, a obesidade pode estar relacionada a fatores psicológicos como o controle, a ansiedade e o desenvolvimento emocional. Desta forma, faz-se necessário uma investigação sistemática, quando se propõe construir conhecimentos que possam subsidiar uma prática de assistência.

Uma forma de avaliar a percepção das pessoas sobre o controle que exercem em relação a even- tos de suas vidas, baseia-se nas formulações teóricas de Rotter (1966). Este autor propôs o construto locus de controle, no qual são identificadas duas tendências - a interna e a externa. As pessoas com locus de controle interno tendem a atribuir a si mesmas o controle, enquanto que os externamente orientados tendem a atribuir a outras pessoas ou ao meio ambiente o controle sobre o que ocorre em suas vidas.

Do ponto de vista operacional, a presença desses componentes da personalidade tem sido avaliada através de escalas. Estes são instrumentos de auto-relato em que o sujeito lê alternativas e avalia se concorda ou não com elas e em que grau. As respostas são avaliadas e traduzidas em escores.

Stotland e Zuroff (1990) verificaram, em muitas pesquisas, uma significativa relação entre locus de controle e perda de peso. No estudo de Aldersberg e Mayer (1949), verificou-se que indivíduos com excesso de peso possuíam uma tendência à externalidade. Para Rotter (1966), locus de controle interno é um potencial preditor para sucessos em programas de perda de peso. Contudo, pesquisas posteriores tanto validaram tal afirmação (Balch \& Ross, 1975; Ross, Kalucy \& Morton, 1983), quanto a contrariaram (Gormally, Rardin \& Black, 1980; Tobias \& MacDonald, 1977). Entretanto, estes estudos foram realizados com adultos. Não foram encontrados trabalhos que relacionassem locus de controle e obesidade infantil.

Outro fator bastante citado como presente na dinâmica da personalidade do indivíduo obeso é a ansiedade. Andrade e Gorenstein (1998), afirmam que este é um estado emocional com componentes psicológicos e fisiológicos, que faz parte do espectro normal das experiências humanas, sendo propulsora do desenvolvimento. Ela pode tornar-se patológica quando é desproporcional à situação que a desencadeia, ou quando não existe um objeto específico ao qual se direcione.

O estudo sobre a ansiedade, do ponto de vista psicológico, salienta uma diferenciação quanto à forma com que ela se apresenta - ansiedade estado e traço. A ansiedade estado é conceituada como um estado emocional transitório ou condição do organismo humano, caracterizada por sentimentos desagradáveis de tensão e apreensão, conscientemente percebidos e por um aumento na atividade do siste- 
ma nervoso autônomo. Os escores de ansiedade/estado podem variar em intensidade de acordo com o perigo percebido e flutuar no tempo (Andrade \& Gorenstein, 1998).

Já ansiedade traço, segundo esses autores, refere-se a diferenças individuais relativamente estáveis na propensão à ansiedade, isto é, a diferenças na tendência de reagir a situações percebidas como ameaçadoras com intensificação do estado de ansiedade.

Os escores de ansiedade traço sã̃ menos suscetíveis a mudanças decorrentes de situações ambientais e permanecem relativamente constantes no tempo.

Soifer (1987, conforme citado por Andrade, 1995), afirma que um alto nível de ansiedade pode ter como sintoma a obesidade, que possivelmente mascara dificuldades internas, afetivase relacionais, requerendo um tratamento psicológico urgente.

Quando se trata de avaliar crianças e adolescentes sob a perspectiva projetiva, uma técnica de investigação bastante utilizada e que abarca aspectos cognitivos e emocionais tem sido o Desenho da Figura Humana.

Azevedo (2000), comparando os resultados obtidos com a aplicação do Desenho da Figura Humana em 30 crianças obesas e 30 crianças não obesas, encontrou que os primeiros apresentaram mais indicadores emocionais.

Venturini (2000), utilizando o DFH com 15 crianças obesas verificou que suas produções evidenciaram transtornos no esquema corporal, ansiedade, insegurança, insatisfação consigo mesmas e sinais de agressividade, entre outros.

A partir dessas considerações, objetivou-se primeiramente neste trabalho, investigar itens evolutivos e emocionais, locus de controle e ansiedade, tendo como sujeitos pré-adolescentes atendidos no Programa de Atenção Multiprofissional a Obesidade da Universidade de São Paulo no campus de Ribeirão Preto, a fim de melhor compreender seu funcionamento psicológico e orientar futuras intervenções com esta clientela.

A literatura sobre obesidade e os distúrbios alimentares vem focalizando, sobretudo, sujeitos do sexo feminino. Esta ênfase fundamenta-se na perspectiva da evolução negativa para distúrbios mais severos, como anorexia e bulimia em meninas (Candy \& Fee, 1998). Por outro lado a obesidade em meninos também tem aumentado conforme os dados veiculados na Folha de São Paulo (1999). Dessa maneira, julga-se oportuno comparar meninas e meninos obesos quanto aos fatores aqui avaliados, configurando-se assim, um segundo objetivo deste trabalho.

\section{Método}

\section{Sujeitos}

Os sujeitos deste estudo foram 26 pré-adolescentes ( 11 meninas e 15 meninos), com idades entre nove e treze anos, que estão freqüentando da $4^{\mathrm{a}}$ a $7^{\mathrm{a}}$ série do $1^{\circ}$ grau. A maior parte deles pertence a famílias com renda mensal de média a baixa.

Em relação ao peso, todos os sujeitos situaram-se no percentil 95 . Isto significa que, segundo critérios da National Center of Health Statistics, Height and Weight of Youth 12-17 years (1981, apud Must, Dallal \& Dietz, 1991), todos se enquadravam no critério de obesidade.

Estes sujeitos foram encaminhados pelo Programa de Assistência à Saúde do Escolar (PROASE) da Prefeitura Municipal de Ribeirão Preto/SP, ao Programa de Atenção Multiprofissional, que é composto por psicólogos, enfermeiros, nutricionistas, médicos e educadores físicos.

De acordo com a Resolução 196/96 do Ministério da Saúde, os aspectos éticos foram considerados através da obtenção do consentimento livre e esclarecido dos pais para que seus filhos fossem submetidos à avaliação.

\section{Procedimento}

Foram aplicados, coletivamente, três instrumentos, cada um em uma sessão, que teve duração média de 50 minutos. Os sujeitos foram acomodados em mesas individuais em uma ampla sala. A aplicação dos instrumentos foi realizada por duas psicólogas, após três encontros destinados ao estabelecimento do rapport.

Os instrumentos foram aplicados na ordem que se segue: Teste do Desenho da Figura Humana, Escala de Ansiedade "O que Penso e Sinto" e a Es- 


\section{Ana Maria Pimenta Carualho}

cala de Locus de Controle para crianças. Quanto ao Teste do Desenho da Figura Humana, a instrução solicitava que os sujeitos desenhassem, inicialmente, apenas uma pessoa inteira. Posteriormente, era entregue uma nova folha e solicitava-se que fosse desenhado somente uma pessoa do sexo oposto à do primeiro desenho. Foi avaliado apenas o desenho do mesmo sexo da criança. A padronização utilizada para este teste foi a de Hutz e Antoniazzi (1995), com base no esquema de Koppitz (1968). Também foi realizada uma análise qualitativa baseada em Van Kolck (1984). A Escala de Ansiedade "RCMAS" foi desenvolvida por Reynolds \& Richmond (1978) e padronizada por Gorayeb (1994) com o título "O que Penso e Sinto". Compõe-se de 28 frases em que o sujeito deve escolher a resposta sim ou não. Este é um instrumento que propõe medir a ansiedade enquanto traço de personalidade.

A Escala de Locus de Controle construída por Milgram e Milgram (1975) e padronizada por Feres (1981), é composta por 24 itens, que retratam situações de sucesso ou fracasso, com alternativas de múltipla escolha, que vão daquelas que atribuem a responsabilidade do desfecho mais ao próprio sujeito àquelas que a atribuem totalmente ao outro.

\section{Resultados}

Os resultados das avaliações de cada sujeito, deste grupo, foram comparados com os dados normativos dos instrumentos utilizados. Tomou-se como referência as médias e desvios padrão. Analisou-se a distribuição de meninos e meninas separadamente pois este era um dos objetivos do trabalho.

Foi aplicado o Teste Exato de Fisher para avaliar a significância das diferenças entre as distribuições dos escores de meninos e meninas. Esta prova foi escolhida uma vez que as freqüências de ocorrência de sujeitos com escores situados nas posições acima, na média e abaixo eram pequenas.

Iniciando-se a apresentação dos resultados, a Tabela 1 mostra a distribuição dos sujeitos segundo seus resultados na Escala Evolutiva do Teste do Desenho da Figura Humana -DFH.

Tabela 1 - Distribuição dos sujeitos em relação aos escores médios da Escala Evolutiva do DFH.

\begin{tabular}{l|cc|cc|cc}
\hline $\begin{array}{l}\text { Posição em relação } \\
\text { à média }\end{array}$ & \multicolumn{2}{|c|}{ Meninas } & \multicolumn{2}{c|}{ Meninos } & \multicolumn{2}{c}{ Total } \\
& F & $\%$ & F & $\%$ & F & $\%$ \\
\hline Acima & 0 & 0 & 0 & 0 & 0 & 0 \\
\hline na média & 8 & 30,8 & 11 & 42,3 & 19 & 73,1 \\
\hline Abaixo & 3 & 11,5 & 4 & 15,4 & 7 & 26,9 \\
\hline Total & 11 & 42,3 & 15 & 57,7 & 26 & 100 \\
\hline
\end{tabular}

Comparando-se as distribuições de meninos e meninas encontrou-se $p=0,40435$, para $\alpha=0,05$ o que aponta para a não significância da diferença entre as distribuições. Para o grupo como um todo, verificou-se que a maior parte dos sujeitos, $73,1 \%$, situou-se nos valores médios e $26,9 \%$ apresentaram escores que, comparativamente à população, os co- loca abaixo da média. É necessário lembrar que, os dados normativos do DFH não apresentam diferenças entre meninos e meninas. Os valores normais são válidos para ambos os sexos (Hutz \& Antoniazzi, 1995).

Os valores relativos à Escala Emocional estão assinalados na Tabela 2. 
Tabela 2 - Distribuição dos sujeitos em relação aos escores médios da Escala Emocional do DFH.

\begin{tabular}{l|cc|cc|ccc}
\hline $\begin{array}{c}\text { Posição em relação } \\
\text { à média }\end{array}$ & \multicolumn{2}{|c|}{ Meninas } & \multicolumn{2}{c|}{ Meninos } & \multicolumn{2}{c}{ Total } \\
& F & $\%$ & F & $\%$ & F & $\%$ \\
\hline Acima & 0 & 0 & 3 & 11,5 & 3 & 11,5 \\
\hline na média & 9 & 34,6 & 12 & 46,2 & 21 & 80,8 \\
\hline Abaixo & 2 & 7,7 & 0 & 0 & 2 & 7,7 \\
\hline Total & 11 & 42,3 & 15 & 57,7 & 26 & 100 \\
\hline
\end{tabular}

Comparando-se as distribuições de meninos e meninas, foram considerados os valores acima e na média, para uma primeira comparação. Obtevese um valor de $\mathrm{p}=0,22488$ para $\alpha=0,05$. Portanto, as distribuições entre ambos não têm diferença estatisticamente significante.

Comparando-se as ocorrências na média e abaixo, obteve-se uma probabilidade po $=0,35968$, também não significante.

Portanto, conclui-se pela não diferença entre as distribuições de meninos e meninas nas diferentes posições em relação aos valores médios. A maior parte dos sujeitos teve escores compatíveis com as médias de suas faixas etárias. Entretanto, apenas meninos apresentaram sinais indicativos de problemas emocionais.

De acordo com uma análise qualitativa do DFH, baseada na proposição de Van Kolk (1984), apareceram com freqüência indicadores de: dependência materna, dificuldades no contato social, dificuldades em lidar com os sentimentos e com os impulsos sexuais e agressivos, preocupação com uma aparência social aceitável, conflitos em relação à virilidade, à sexualidade e entre autonomia e dependência.

Os resultados exibidos na Tabela 3 mostram uma distribuição relativamente semelhante entre os sujeitos, nas diferentes posições, em relação às medias da Escala de Locus de Controle.

Tabela 3 - Distribuição dos sujeitos em relação aos escores médios da Escala de Locus de Controle.

\begin{tabular}{l|cc|cc|cc}
\hline \multirow{2}{*}{$\begin{array}{c}\text { Posição em relação } \\
\text { à média }\end{array}$} & \multicolumn{2}{|c|}{ Meninas } & \multicolumn{2}{c|}{ Meninos } & \multicolumn{2}{c}{ Total } \\
& F & $\%$ & F & $\%$ & F & $\%$ \\
\hline acima da média & 7 & 26,9 & 5 & 19,2 & 12 & 46,1 \\
\hline na média & 0 & 0 & 2 & 7,7 & 2 & 7,7 \\
\hline abaixo da média & 4 & 15,4 & 8 & 30,8 & 12 & 46,1 \\
\hline Total & 11 & 42,3 & 15 & 57,7 & 26 & 100 \\
\hline
\end{tabular}

Não se verificam tendências à internalidade ou externalidade, neste grupo de sujeitos. Comparando-se as distribuições dos meninos e meninas através do Teste Exato de Fisher (eliminando-se os valores relativos à media) obteve-se $\mathrm{p}=0,2068$ para $\alpha=0,05$. Não foram encontradas diferenças entre as distribuições acima e abaixo da média, de meninos e meninas, a despeito das meninas tenderem à externalidade e os meninos à internalidade.

Em relação à Escala de Ansiedade "O que Penso e Sinto", os sujeitos obtiveram os resultados apresentados na Tabela 4. 
Tabela 4 - Distribuição dos sujeitos em relação aos escores médios da Escala de Ansiedade Infantil "O que Penso e Sinto"

\begin{tabular}{l|cc|cc|cc}
\hline $\begin{array}{c}\text { Posição em relação } \\
\text { à média }\end{array}$ & \multicolumn{2}{|c|}{ Meninas } & \multicolumn{2}{c|}{ Meninos } & \multicolumn{2}{c}{ Total } \\
& F & $\%$ & F & \% & F & $\%$ \\
\hline acima da média & 1 & 3,8 & 0 & 0 & 1 & 3,8 \\
\hline na média & 6 & 23,1 & 9 & 34,6 & 15 & 57,7 \\
\hline abaixo da média & 4 & 15,4 & 6 & 23,1 & 10 & 38,5 \\
\hline Total & 11 & 42,3 & 15 & 57,7 & 26 & 100 \\
\hline
\end{tabular}

Os dados mostram que os sujeitos estão situados dentro da média, exibindo, em sua maioria, níveis de ansiedade esperados para crianças nessas faixas etárias. A comparação entre as distribuições de meninos e meninas, pelo Teste Exato de Fisher (eliminando-se os valores iguais à media), confirmam a hipótese de uma não diferenciação entre eles. Foi obtido um $p=0,5449$ para $\alpha=0,05$.

Segundo os dados obtidos com esta escala, que se propõe a medir a ansiedade enquanto traço de personalidade, apenas $3,8 \%$ dos sujeitos apresentaram sinais de ansiedade aumentada, 38,5\% obtiveram escores compatíveis com ansiedade reduzida e $57,7 \%$,dentro do esperado para o sexo e idade.

A seguir são apresentados os dados relativos à Escala da Mentira. Esta é uma sub escala da Escala Ansiedade Infantil "O que Penso e Sinto" e propõese a avaliar o quanto o sujeito responde de acordo com o que ele pensa que se espera dele, no sentido de mostrar uma imagem positiva de si mesmo.

Tabela 5 - Distribuição dos sujeitos em relação aos escores médios da Sub-Escala da Mentira da Escala de Ansiedade Infantil "O que Penso e Sinto"

\begin{tabular}{l|cc|cc|cc}
\hline $\begin{array}{c}\text { Posição em relação } \\
\text { à média }\end{array}$ & \multicolumn{2}{|c|}{ Meninas } & \multicolumn{2}{c|}{ Meninos } & \multicolumn{2}{c}{ Total } \\
& F & $\%$ & F & \% & F & $\%$ \\
\hline acima da média & 1 & 3,9 & 2 & 7,6 & 3 & 11,5 \\
\hline na média & 9 & 34,6 & 10 & 38,5 & 19 & 73,1 \\
\hline abaixo da média & 1 & 3,9 & 3 & 11,5 & 4 & 15,4 \\
\hline Total & 11 & 42,4 & 15 & 57,6 & 26 & 100 \\
\hline
\end{tabular}

Verificou-se que a maior parte dos sujeitos, $73,1 \%$, situou-se na faixa correspondente aos valores médios. Portanto, a maioria apresentou resultados que estão dentro do esperado, de acordo com as normas de padronização do instrumento.

Comparando-se as distribuições de meninos e meninas não se verificaram diferenças estatisticamente significantes, através do teste Exato de Fisher. Eliminando-se os valores acima da média, obteve-se $p=0,40370$ para $\alpha=0,05$. Eliminando-se os valores abaixo da média, obteve-se $p=0,5714$ para $\alpha=0,05$.

\section{Discussão}

Com base no que a literatura vem mostrando acerca das relações entre aspectos da personalidade e obesidade, este trabalho propôs-se a avaliar um grupo de pré-adolescentes obesos - meninos e meni- 
nas - e verificar as tendências evolutivas, emocionais, de locus de controle e de ansiedade traço apresentadas pelo grupo.

Embora com limitações, dentre as quais destacam-se o número reduzido de sujeitos e a ausência de um grupo controle, as questões formuladas puderam ser respondidas.

Com relação aos aspectos evolutivos e emocionais, avaliados pelo DFH, não se verificou uma tendência do grupo a diferenciar-se da população geral de pré-adolescentes.

Entretanto, alguns indivíduos apresentaram sinais de imaturidade e indicadores de problemas emocionais.

Do ponto de vista dos aspectos evolutivos, meninos e meninas não se diferenciaram. Os dois subgrupos exibiram resultados compatíveis com a média esperada e alguns indivíduos exibiram resultados inferiores aos esperados para suas faixas etárias. Entretanto quanto aos aspectos emocionais, verificou-se uma tendência dos meninos a exibir mais sinais emocionais que as meninas.

Tais resultados diferenciam-se dos de Azevedo (2000), que encontrou através do DFH, mais sinais de problemas emocionais num grupo de crianças obesas, comparadas com um grupo de não obesas. No que tange à identificação de problemas emocionais, numa análise qualitativa das produções do DFH, verificaram-se sinais semelhantes aos encontrados por Venturini (2000).

A literatura sobre obesidade e distúrbios alimentares tende a focalizar mais as mulheres e meninas adolescentes, sobretudo, porque os estudos vêm demonstrando uma evolução negativa para distúrbios mais severos como a bulimia e a anorexia, cuja prevalência é maior em mulheres (Candy \& Fee, 1998).

Outro fator que parece relacionar-se à pouca atenção dada aos meninos foi mostrado no trabalho de Pierce e Wardle (1993) quanto à apreciação dos pais com relação à imagem corporal de seus filhos e sua relação com a massa corporal e a auto estima. Estes pesquisadores encontraram que, para os meninos, uma baixa auto-estima esteve relacionada com o sentir-se magro e ser avaliado como magro. Para as meninas esta associação se faz com o ser gorda.

O presente estudo, no entanto, apresentou al- guma evidência de problemas emocionais nos meninos, ocorrendo de forma concomitante ao excesso de peso. Esta observação justifica esclarecimentos e atenção a esta clientela que, tende a constituir-se na maior parte da população de crianças encaminhadas a serviços de saúde mental (Silvares, 1993.)

Quanto ao locus de controle, não se verificou tendência à internalidade ou externalidade. Há variações individuais e, de modo geral, um equilíbrio na identificação de sujeitos em uma e outra categoria.

Isto já foi objeto de investigação em adultos, na década de 70, e para este grupo também não foram encontradas tendências claras à externalidade (Gormanous \& Lowe, 1975).

A investigação sobre a tendência do locus de controle constituiu-se numa forma de operacionalizar a investigação sobre uma crença de que as pessoas obesas têm pouco controle sobre si e suas vidas: Nessa linha de raciocínio, Stotland e Zuroff (1990) propuseram que a avaliação do locus de controle geral não discrimina obesos e não obesos. Para estes pesquisadores, deve-se buscar avaliar essa variável em relação ao comportamento alimentar. Eles propuseram uma escala para a situação de alimentação a ser utilizada com adultos. Entretanto, seria necessário adaptar essa escala para crianças e pré-adolescentes pois, ela é útil na previsão do engajamento das pessoas a programas de reeducação e modificação do comportamento alimentar, com vistas à perda de peso.

Quanto à ansiedade, encontrou-se que a maior parte do grupo apresentou escores abaixo do esperado para a população desta faixa etária. Seria essa uma tendência: apresentar níveis reduzidos de ansiedade enquanto traço?

Pode-se supor, como Kahtalian (1992), que a obesidade tem em si a função de conter toda a ansiedade e angústia não permitindo sua manifestação através de um instrumento de auto relato?

Os resultados obtidos através da Escala da Mentira permitem dizer que as crianças responderam de forma adequada. Infere-se, portanto, que não ofereceram respostas que julgavam socialmente corretas e aceitas. Entretanto há, ainda, que lembrar que os resultados mostram variações individuais e alguns sujeitos mostram sinais mais evidentes de ansiedade.

Finalizando, os resultados parecem sugerir 
que não há, em relação a traços mais estáveis de personalidade, como o locus de controle, tendência unilateral para externalidade ou internalidade.

Quanto à ansiedade encontrou-se uma proporção de sujeitos com níveis abaixo da média, embora a maior parte esteja apresentando resultados compatíveis com o esperado para sua faixa etária.

De outro lado, utilizando o DFH, que apreende aspectos situacionais, verificou-se sinais de imaturidade emocional, como manifestação atual na dinâmica intrapsíquica, também para alguns indivíduos.

Acredita-se que tais resultados estejam indicando que nem sempre ser gordo se faz acompanhar de traços de personalidade que definam um perfil, a despeito de alguns indivíduos estarem apresentando, no momento da avaliação, sinais indicativos de problemas emocionais.

Pode-se concluir neste estudo, que não há diferenças entre pré-adolescentes obesos e não obesos quanto às variáveis medidas. Ainda, no âmbito deste trabalho, não se verificaram diferenças entre meninos e meninas. Contudo, chamou a atenção os dados relativos à presença de meninos com indicadores emocionais acima do esperado e a ausência deles para as meninas. Isto sugere a necessidade de maior atenção aos meninos em relação às condutas alimentares. Além disso, verificou-se que alguns indivíduos apresentaram sinais de sofrimento psicológico que demandaria atenção. Considera-se que estudos desta natureza sejam relevantes para elucidar aspectos relativos ao funcionamento psicológico de pré-adolescentes obesos, tendo em vista os índices crescentes de obesidade e seu difícil manejo.

\section{Referências Bibliográficas}

Aldersberg, D. \& Mayer, M. (1949). Results of prolonged medical treatment of obesity with diet alone, diet and thyroid preparations, and diet and amphetamine. Journal of Clinical Endocrinology, 9, 275-284.

Andrade, T.M. (1995). Estudo psicológico de crianças e adolescentes obesos. Em Fisberg, M. (Org.), Obesidade na infância e adolescência (pp.100104). São Paulo: Fundação BYK.

Andrade, L.H.S.G., \& Gorestein, C. (1998). Aspec- tos gerais das escalas de avaliação de ansiedade. Revista de Psiquiatria Clínica, 25, n.6, p.285290.

Azevedo, M.A.S.B. (2000). Obesidade na infância: visão psicológica [Resumo]. Em José Onildo B. Contel (Org.). Anais do $8^{\circ}$ Ciclo de estudos em saúde mental (p.280), Ribeirão Preto: Pós-Graduação em Saúde Mental - FMRP-USP.

Balch, P. \& Ross, A.W. (1975). Predicting success in weight reduction as a function of locus of control: a unidimensional and multidimensional approach. Journal of Consulting and Clinical Psychology, 43, 119.

Campos, A.L.R. (1993). Aspectos psicológicos da obesidade. Pediatria Moderna, 29 (2), 129-133.

Candy, C.M. \& Fee, V.E. (1998) Underlying Dimensions and Psychometric Properties of the Eating Behaviors and Body Image Test for Preadolescent Girls. Journal of Clinical Child Psychology,27, n.1, p,117-127.

Escrivão, M.A.M.S. \& Lopez, F.A. (1995). Prognóstico da Obesidade na Infância e na Adolescência. Em Fisberg, M. (Org.), Obesidade na infância e adolescência (pp.146-148). São Paulo: Fundação BYK. Cap.2.

Feres, N.A.L. (1981). Locus de controle e comparação social na atribuição de causalidade por crianças. Tese de Doutorado Não-Publicada, Fundação Getúlio Vargas. Rio de Janeiro, RJ.

Fisberg, M. (1995). Obesidade na Infância e na Adolescência. Em M. Fisberg (Org.), Obesidade na infância e adolescência (pp. 9-13). São Paulo: Fundação BYK.

Gigliotti, F. (1999, fev.22). Obesidade atinge $25 \%$ dos jovens. Folha de São Paulo, p.7.

Gorayeb, M.A.M. (1994).Adaptação, normatização $e$ avaliação das qualidades psicométricas da RCMAS para uma amostra de escolares de oito a treze anos de Ribeirão Preto. Dissertação de Mestrado Não-Publicada, Universidade de São Paulo. Ribeirão Preto, SP.

Gormally, J., Rardin, D., \& Black, S. (1980). Correlate of successful response to a behavioral 
weight control clinic. Journal of Counseling Psychology, 27, 179-191.

Gormanous, G.K. \& Lowe, W.C. (1975). Locus of control and obesity. Psychological Reports, 37, 30.

Hutz C.S. \& Antoniazzi, A.S. (1995). O desenvolvimento do desenho da Figura Humana em crianças de 5 a 15 anos de idade: normas para avaliação. Psicologia: Reflexão e Crítica, 8 (1), 318.

Kahtalian, A. (1992). Obesidade: um desafio. Em J. de Mello Filho (Org.), Psicossomática hoje (pp. 273-278). Porto Alegre: Artes Médicas.

Koppitz, E.M. (1968). Psychological evaluation of children's human figure drawings. New York, NY: Grune \& Stratton.

Milgram, N.A. \& Milgram, R.M. (1975). Dimensions of locus de control in children. Psychological Reports, 37, 523-538.

Must, A., Dallal, G.E. \& Dietz, W.H. (1991). Reference data for obesity 85 th and $95^{\text {th }}$ percentiles of Body Mass Index - a correction. Am J Clin Nutrition, 54, 773.

Pierce, J.W. \& Wardle, J. (1993). Self-esteem, parent appraisal and body size in children. Journal of Child Psychology and Psychiatry and allied Disciplines, v.3, n.7, p.1125-1135.

Reynols, C.R. \& Richmond, B.O. (1978). What I Think and Feel: a revised measure of children's manifest anxiety. Journal of Abnormal Child Psychology, 6 (2), 271-280.

Ross, M., Kalucy, R.S. \& Morton, J.E. (1983). Locus of control in obesity: predictors of success in a jaw-wiring programme. British Journal of Medical Psychology, 53, 49-56.

Rotter, J.B. (1966). Generalized expectancies for internal versus external control of reinforcement. Psychological Monographs, 80, n.1. (Whole n. 609).

Silvares, E.F.M. (1993). O papel preventivo das clínicas-escola de psicologia em seu atendimento a crianças._Temas em Psicologia, 2, p.87-97.
Stotland, S.S. \& Zuroff, D.C. (1990). A new measure of weight locus of control: the dieting beliefs scale. Journal of Personality Assessment, 54 (1,2), 191-203.

Tobias, L.L. \& MacDonald, M.L. (1977). Internal locus of control and weight loss: an insufficient condition. Journal of Consulting and Clinical Psychology, 45, 647-653.

Van Kolk, O.L. (1984). Testes projetivos gráficos no diagnóstico psicológico. São Paulo: EPU. (Temas Básicos de Psicologia, v.5).

Venturini, L.P. (2000). Obesidade e família - uma caracterização de famílias de crianças obesase a percepção dos familiares e das crianças de sua imagem corporal. Dissertação de Mestrado, Universidade de São Paulo, Ribeirão Preto. 